\title{
INFLUENCIA DE LOS HERMANOS EN EL COMPORTAMIENTO SEXUAL DE RIESGO Y PREVENTIVO: UNA REVISIÓN
}

\author{
David Javier Enríquez-Negrete y Ricardo Sánchez-Medina \\ UNAM, FES IZTACALA
}

MÉXICO

\begin{abstract}
RESUMEN
El presente estudio tuvo como objetivo hacer una revisión de la literatura especializada publicada entre 1990 y 2014 relativa a la influencia fraterna en el comportamiento sexual; para ello se utilizó como metodología los núcleos temáticos, los cuales fueron: socialización sexual fraterna e influencia de los hermanos sobre el comportamiento sexual de riesgo/prevención. Como resultado de esta revisión se encontró que los hermanos son una fuente de socialización sexual secundaria y la estructura familiar tiene una estrecha relación con las creencias, actitudes e intenciones que los hermanos mayores transmiten a los menores sobre el sexo. Hay una tendencia a utilizar la teoría del aprendizaje social para explicar la influencia fraterna y de conceptuar a los hermanos mayores como agentes nocivos para el comportamiento sexual de los hermanos menores. Condiciones como la cercanía, el rol fraterno (mayor o menor) y el género, juegan un papel fundamental en la compresión de cómo los hermanos mayores influyen en el comportamiento sexual de los menores. Para finalizar, en la discusión se abordan del estado actual y las líneas futuras de investigación sobre este tema.
\end{abstract}

Palabras Clave:

Socialización, hermanos, VIH/SIDA, ITS, embarazo, familia

\section{SIBLINGS'S INFLUENCE IN SEXUAL RISK BEHAVIOR AND PREVENTION: A REVIEW}

\begin{abstract}
Objective. Reviewing the literature published between 1990-2014 on the fraternal influence on sexual behavior. Methodology. The core themes were: fraternal sexual socialization and sibling influence on sexual behavior risk/prevention. Results. The siblings are a source of secondary sexual socialization and family structure are closely linked to the beliefs, attitudes and intentions that older siblings transmitted to children about sex. There is a tendency to use social learning theory to explain the influence fraternal and conceptualize older siblings as harmful to the sexual behavior of younger siblings. Conditions such as proximity, fraternal role (younger or older) and gender are key in understanding how older brothers influence the sexual behavior. Discussion. In terms of the current state and future research on this topic.
\end{abstract}

Keywords:

Socialization, siblings, HIV/AIDS, STIs, pregnancy, family

Bitácora del Artículo:

| Recibido: 5 de Diciembre de 2015 | Aceptado: 5 de Enero de 2015 | Publicado en línea: Enero-Junio de 2016 | 
Revisión de literatura | Influrncia de los hermanos en el comportamiento sexual ... E Enriquez-Negrete eZ Sánchez-Medina

\title{
Autoría y Derechos de Propiedad Intelectual
}

\section{INFLUENCIA DE LOS HERMANOS EN EL COMPORTAMIENTO SEXUAL DE RIESGO Y PREVENTIVO: UNA REVISIÓN}

\author{
David Javier Enríquez-Negrete y Ricardo Sánchez-Medina
}

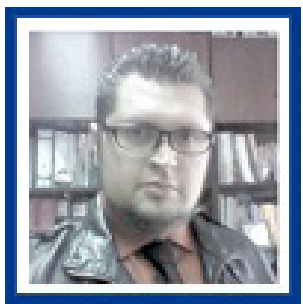

David Javier Enriquez Negrete

FESI UNAM

Correo: david.enriquez@ired.unam.mx

Doctor en Psicología por la UNAM en el área de Psicología y Salud. Tutor en educación e-learnig por la UNAM. Profesor Asociado C Tiempo Completo de la Carrera de Psicología en el Sistema de Universidad Abierta y Educación a Distancia (SUAyED-UNAM).

Ver más...

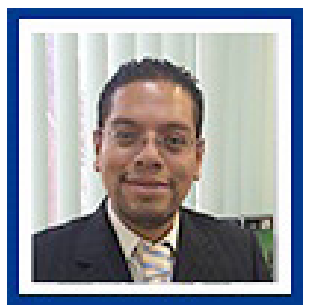

Ricardo Sánchez Medina

FESI UNAM

Correo: risame81@gmail.com

Doctor en Psicología en la UNAM en el campo de Psicología y Salud. Profesor de Asignatura A en la FESI-UNAM en la carrera de Psicología en el Sistema de Universidad Abierta y Educación a Distancia (SUAyED) en los Programa de Profundización en Psicología Clínica y de Profundización en Psicología de la Salud. Ver más...

\section{CONTRIBUCIÓN DE LOS AUTORES}

El primer autor estuvo a cargo de la coordinación del trabajo, la idea original y argumentación del estudio, así como de la búsqueda y organización de la información, análisis de datos, redacción de la integración para resultados y la conclusión. | El segundo autor contribuyó en la estructura metodológica, búsqueda y organización de la información por núcleos temáticos, revisión de estilo, análisis de datos, integración para resultados e introducción.

\section{AgRADECIMIENTOS}

Este estudio forma parte de un proyecto más general "Impacto del contexto familiar en el comportamiento sexual preventivo de jóvenes universitarios" financiado por el Programa de Apoyo a Proyectos de Investigación e Innovación Tecnológica (PAPIIT-UNAM) con clave IA301616

\section{Datos de Filiación de los Autores}

\author{
SUAyED,FES Iztacala, UNAM,
}

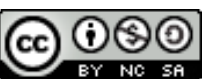

Copyright: (c) 2016 Enriquez-Negrete, D.J. \& Sánchez-Medina, R.

Este es un artículo de acceso abierto distribuido bajo los términos de la licencia Creative Commons Reconocimiento-NoComercial-Compartirlgua 4.0 Internacional, por lo que su contenido gráfico y escrito se puede compartir, copiar y redistribuir total o parcialmente sin necesidad de permiso expreso de sus autoras con la única condición de que no se puede usar con fines directamente comerciales y los términos legales de cualquier trabajo derivado deben ser los mismos que se expresan en la presente declaración. La única condición es que se cite la fuente con referencia a la Revista Digital Internacional de Psicología y Ciencia Social y a sus autoras. 


\section{TABLA DE CONTENIDO}

INTRODUCCIÓN

MÉTOdo

Rango de la búsqueda, 5

Unidades de análisis, 5

Núcleos temáticos, 5

Artículos encontrados, 5

Composición de la muestra de artículos, 5

Criterio de inclusión y exclusión de artículos, 5

Procedimiento, 6

RESULTADOS

a) Socialización sexual fraterna, 6

b) Influencia de los hermanos sobre el comportamiento sexual de riesgo y prevención, 7

Discusión 


\section{INTRODUCCIÓN}

a investigación desarrollada en el terreno de la salud sexual se ha encaminado hacia la identificación de los factores que hacen más probable que las personas tengan conductas sexuales saludables y preventivas (Noar, 2007) para evitar el contagio de alguna Infección de Transmisión Sexual (ITS), incluido el VIH/SIDA, o tener un embarazo no planeado (Bárcena, Rendón \& Robles, 2011). Tanto las ITS como los embarazos no planeados constituyen un problema serio de salud (Secretaría de Salud, 2002; Consejo Nacional para la Prevención y Control del SIDA [CENSIDA], 2011; 2012) y un aspecto de atención prioritaria entre los adolescentes mexicanos (Encuesta Nacional de Salud y Nutrición [ENSANUT], 2012).

De acuerdo con el meta-análisis realizado por Kotchick, Shaffer, Miller, \& Forehand (2001), se han logrado avances significativos en la identificación de variables predictoras de diversos comportamientos sexuales preventivos y de riesgo, aunque señalan que la mayor parte de la literatura especializada en esta área recae sobre el estudio de aquellas variables centradas en el individuo (intenciones para usar preservativo, actitudes hacia el condón, habilidades de comunicación sexual y de negociación para utilizar preservativo, por citar algunos ejemplos). La conclusión de Kotchick, et al. (2001), después de haber revisado más de 500 artículos especializados, fue que se le ha brindado poca atención a los factores del contexto familiar que influyen en el comportamiento sexual.

En este sentido, las investigaciones centradas en describir los factores del contexto familiar que influyen en el comportamiento sexual de los adolescentes, se centran principalmente en evaluar variables parentales (Hovell, et al., 1994; Miller, Forehand, \& Kotchick, 1999; Upchurch, Aneshensel, Sucoff, \& Levy-Storms, 1999; Davis, \& Friel, 2001; Vélez-Pastrana, González-Rodríguez, \& Borges-Hernández, 2005; Uribe, 2007; Vukovic, \& Bjegovic, 2007; Palacios, 2009; Manlove, Wildsmith, Ikramullah, Terry-Humen, \& Schelar, 2012; Oliveira-Campos, Giatti, Malta, \& Barreto, 2013) y dejan en un segundo plano otras fuentes de influencia familiar como por ejemplo, los hermanos. Widmer (1997) señala que si bien los padres son la fuente primaria de socialización sexual en los hijos, la influencia de los hermanos también debe de considerarse por lo significativo que llegan a ser las relaciones fraternas.
Las relaciones fraternas son centrales en la vida de una persona, debido a su larga duración -del nacimiento a la muerte- (Lucey, 2010), y porque los hermanos comparten las mismas experiencias familiares y una herencia genética, social y cultural común (Goetting, 1986). En algunos países como México, los hermanos fungen como cuidadores secundarios (Cicirelli, 1994), principalmente cuando ambos padres tienen que trabajar, lo cual favorece que pasen mucho tiempo juntos en comparación con los propios padres; además de que al cohabitar en la misma vivienda pueden pasar la mayor parte del día juntos (Diop-Sidibe, 2005). De hecho, en ocasiones los hermanos llegan a tomar tanta responsabilidad en torno al cuidado de sus hermanos que se convierten en sustitutos de los padres; sobre todo en los casos en los cuales los papás tienen una participación y compromiso nulo o deficiente con los hijos (Goetting, 1986).

Asimismo, las relaciones entre hermanos pueden llegar a ser más significativas para sociedades con valores colectivistas, donde la lealtad, el auto sacrificio y la cooperación son atributos característicos de las relaciones interpersonales (Triandis, 2006; Triandis, \& Suh, 2002). Estos valores promovidos en países como México pueden nutrir y mantener los atributos que caracterizan las relaciones fraternas, como por ejemplo, el amor, la cercanía (Buist, Deković, \& Prinzie, 2013), la confianza, el respeto, el apoyo, los secretos y la amistad (Enríquez, Sánchez, \& Robles, 2011), de tal forma que estas cualidades coadyuvan al fortalecimiento del vínculo afectivo fraterno. De acuerdo con Buist, et al. (2013) y Feinberg, \& Hetherington (2000), cuando las relaciones entre hermanos son cercanas se favorece la influencia de un hermano sobre otro.

Bajo este marco se torna comprensible por qué los hermanos influyen en el ajuste psicosocial, en la relación con los amigos, con la pareja, así como en los comportamientos saludables y de riesgo (Feinberg, et al., 2013).

De esta forma, después de revisar las evidencias empíricas expuestas anteriormente, no resulta insensato pensar en los hermanos como agentes de socialización sexual, los cuales pudieran tener influencia en alguno o varios comportamientos sexuales de riesgo o preventivos. Sin embargo, la conclusión del meta-análisis realizado por Buist, et al. (2013) y la revisión de Feinberg, et al. (2013) versan sobre la escasa atención puesta al estudio de las relaciones entre hermanos a pesar del gran impacto que pueden tener en el comportamiento, incluso en el sexual.

Evaluar la influencia de los hermanos en el comportamiento sexual preventivo y de riesgo ofrece una perspectiva más amplia sobre el complejo proceso de 
socialización sexual que brinda la familia, favoreciendo una visión incluyente más allá de los padres como agentes de socialización. Asimismo, se podría delimitar sí es que los hermanos tienen un efecto directo sobre el comportamiento sexual, sobre variables psicosociales o conductuales predictoras del comportamiento sexual preventivo y de riesgo, pero principalmente describir los efectos combinados del grupo de padres y hermanos en relación al comportamiento sexual (Enríquez, 2014).

Por tanto, el propósito de este artículo fue revisar la literatura especializada publicada entre 1990 y 2014 relativa al papel que tienen los hermanos en la socialización sexual fraterna, con el objetivo de describir el estado actual de este tópico y de analizar a través de la evidencia empírica publicada, cómo los hermanos influyen en el comportamiento sexual de riesgo y preventivo.

\section{MÉTodo}

\section{Rango de la búsqueda}

Con el objetivo de buscar y reunir la mayor cantidad de artículos posibles para realizar la presente revisión de la literatura, se buscaron publicaciones fechadas entre 1990 y 2014.

\section{Unidades de análisis}

Las unidades de análisis fueron cuatro, las cuales se seleccionaron a partir de la relevancia científica y académica, así como del área disciplinar. Las unidades fueron las siguientes: 1) ScienceDirect -ElSevier, con acceso a 1800 revistas científicas con más de 9 millones de artículos (ElSevier, 2011); 2) JSTORE, con acceso a 2000 revistas académicas que se ofrecen para consulta en más de 160 países; 3) PsycINFO, con cerca de 2500 revistas donde se publican artículos dictaminados por pares de expertos en las ciencias de la conducta (American Psychological Association, 2014); y 4) MEDLINE, la cual depende de la Biblioteca Nacional de Medicina de los Estados Unidos y abarca entre sus áreas las ciencias del comportamiento (United States National Library of Medicine, 2014).

\section{Núcleos temáticos}

Fueron dos los núcleos temáticos de análisis: 1) socialización sexual fraterna y 2) influencia de los hermanos sobre el comportamiento sexual de riesgo y prevención. Los descriptores utilizados para realizar la búsqueda en las unidades de análisis fueron: a) sibling influences on sexual behavior, b) siblings on initiation of sexual intercourse, c) influence of older siblings on sexual behavior, y d) sexual behavior sibling.

\section{Artículos encontrados}

La Tabla 1 muestra el total de artículos encontrados en cada una de las bases de datos por cada descriptor utilizado.

\section{Tabla 1.}

Total de artículos encontrados por unidad de análisis y descriptor

\begin{tabular}{|c|c|c|c|c|}
\hline \multicolumn{5}{|c|}{ NúMERO de ARTÍCULOS POR UNIDAD DE ANÁLISIS* } \\
\hline DESCRIPTORES & ScienceDirect & JSTORE & PsycINFO & MEDLINE \\
\hline $\begin{array}{l}\text { Sibling } \\
\text { influences } \\
\text { on sexual } \\
\text { behavior }\end{array}$ & 178 & 11938 & 96 & 32 \\
\hline $\begin{array}{l}\text { Siblings on } \\
\text { initiation } \\
\text { of sexual } \\
\text { intercourse }\end{array}$ & 44 & 15218 & 2296 & 3 \\
\hline $\begin{array}{l}\text { Influence } \\
\text { of older } \\
\text { siblings } \\
\text { on sexual } \\
\text { behavior }\end{array}$ & 100 & 11965 & 31691 & 8 \\
\hline $\begin{array}{l}\text { Sexual } \\
\text { behavior } \\
\text { sibling }\end{array}$ & 154 & 9992 & 14 & 234 \\
\hline $\begin{array}{l}\text { Total de } \\
\text { artículos }\end{array}$ & 476 & 49113 & 34097 & 277 \\
\hline $\begin{array}{l}\text { *El número de } \\
\text { no son mutuar } \\
\text { pudo encontrar } \\
\text { repetirse en la }\end{array}$ & $\begin{array}{l}\text { tículos encontrad } \\
\text { ente excluyentes, } \\
\text { e en más de una } \\
\text { úsqueda en más d }\end{array}$ & $\begin{array}{l}\text { on en cada ba } \\
\text { lo cual imp } \\
\text { ase de datos } \\
\text { e un descript }\end{array}$ & $\begin{array}{l}\text { se de datos y } \\
\text { ica que el } n \\
\text { y una misma } \\
\text { r. }\end{array}$ & $\begin{array}{l}\text { or descriptor } \\
\text { smo artículo } \\
\text { ntrada pudo }\end{array}$ \\
\hline
\end{tabular}

\section{Composición de la muestra de artículos}

La muestra de artículos seleccionados para la presente revisión fueron 20, los cuales se obtuvieron de las siguientes unidades de análisis: a) ScienceDirect -EISevier, dos artículos; b) JSTORE, nueve artículos; c) PsycINFO, dos artículos; y d) MEDLINE, siete artículos.

\section{Criterio de inclusión y exclusión de artículos}

Para la presente revisión se incluyeron los artículos que cumplían con: a) evaluar al menos una variable asociada al comportamiento sexual de riesgo o preventivo; b) incluir como mínimo, una medida relativa al comportamiento de los hermanos o referente a alguna cualidad de las relaciones fraternas, y c) ser de carácter empírico. Por otro lado, los criterios de exclusión fueron: a) tener fecha de publicación previa al año 1990 y b) ser artículo teórico entre los cuales se incluyeron revisiones de la literatura, capítulos de 
libro y disertaciones, debido a que estos documentos no pasaron por el proceso de evaluación por pares.

\section{Procedimiento}

Los artículos seleccionados para la presente revisión teórica fueron organizados a partir de dos núcleos temáticos. En el primero se agruparon todos los contenidos relativos a la socialización sexual fraterna dispuestos de la siguiente manera: a) estrategias de socialización sexual entre hermanos y b) teorías utilizadas para explicar el proceso de influencia fraterna en el comportamiento sexual. El segundo núcleo temático agrupó los contenidos relativos a la influencia que los hermanos ejercen en el comportamiento sexual de riesgo o preventivo a partir de las siguientes temáticas: a) comportamientos sexuales de riesgo, b) comportamiento sexual preventivo y c) influencia de los hermanos en el comportamiento sexual por diadas fraternas mixtas o del mismo sexo.

\section{Resultados}

A continuación se describen los resultados organizados a partir de los dos núcleos temáticos propuestos para el análisis de la evidencia empírica: a) socialización sexual fraterna y b) influencia de los hermanos sobre el comportamiento sexual de riesgo y prevención.

\section{a) Socialización sexual fraterna}

La socialización sexual es el proceso mediante el cual los conocimientos, actitudes y valores sobre la sexualidad se adquieren. Se trata de un proceso complejo y multidimensional, el cual ocurre gradualmente a lo largo de muchos años e implica la vinculación de mensajes provenientes de diversas fuentes de información. Los mensajes -directos o indirectos- recibidos cubren un amplio rango de temas, los cuales van desde los aspectos biológicos de la reproducción hasta tópicos referidos a valores y actitudes sobre las citas, el sexo, el amor y las relaciones románticas (Ward, 2003).

En el caso de los hermanos mayores, éstos se constituyen como agentes socializadores debido a que son una fuente de información para los hermanos menores respecto a las citas y al comportamiento sexual (Wallace, 2008). El estudio de Ybarra, Emenyonu, Nansera, Kiwanuka, \& Bangsberg (2008) muestra que los hermanos informan a sus hermanos menores sobre la salud sexual en general y específicamente sobre el VIH/SIDA, después de los padres y maestros. Estos mensajes principalmente se llevan a cabo de forma directa a través de la comunicación sexual fraterna, por tanto, hablar, discutir y brindar información a los hermanos sobre sexo puede ser más cómodo (cf. Wallace, 2008), debido a que no existe una barrera generacional, como en el caso de la comunicación entre padres e hijos. Además al no existir esta barrera se favorecen las condiciones para compartir experiencias y opiniones similares, siempre y cuando no exista mucha diferencia de edades entre los hermanos (Kowal, \& Blinn-Pike, 2004).

Para favorecer este proceso de comunicación sexual fraterna se torna indispensable la cercanía entre hermanos (también Ilamada calor/afecto). Kowal, \& Blinn-Pike (2004) describieron como la percepción de una relación estrecha con los hermanos es una condición necesaria para poder hablar entre ellos sobre diferentes temas relativos al sexo. Incluso autores como Martino, et al. (2008) señalan que en las familias el nivel de comunicación es un indicador del grado de afecto entre sus miembros. Además la percepción de cercanía fraterna favorece el involucramiento de los hermanos mayores con los menores, al grado que buscan protegerlos de algún riesgo sexual potencial, por tanto, brindan información y consejos para prevenirlos (Kowal, \& Blinn-Pike, 2004) pero además, dicho involucramiento fraterno también influye en las actitudes que promueven el cuidado de la salud sexual (McHale, Bissell, \& Kim, 2009).

Si bien la comunicación sexual fraterna es una estrategia de socialización sexual directa de los hermanos mayores hacia los menores, el análisis de la literatura muestra que también se puede aprender sobre aspectos sexuales a partir de la imitación y la observación de un modelo -hermano mayor-.

De acuerdo a la revisión realizada, cuando se perciben relaciones estrechas y positivas de cercanía con los hermanos mayores, se generan oportunidades para observar y aprender a través de la interacción y la imitación (Buist, et al., 2013). Este tipo de situaciones favorecen condiciones para que surjan similitudes de cogniciones y comportamientos entre hermanos debido a la influencia del hermano mayor hacia el menor (Feinberg, \& Hetherington, 2000), pero cuando hay rencor fraterno, la conducta modelada tiene menor probabilidad de adoptarse (Brody, 1998).

Bajo estos supuestos, en la mayor parte de los artículos revisados para esta investigación se encontró que la teoría del aprendizaje social es la que principalmente se utiliza para explicar el proceso de influencia fraterna en el comportamiento sexual y contestar a la pregunta “¿cómo los hermanos influyen en la transmisión de aspectos sexuales normativos?" Sin embargo, cabe destacar que de los 20 artículos incluidos en la muestra para la presente revisión, solamente dos buscan evaluar los procesos de influencia basados en esta teoría (McHa- 
le, Bissell, \& Kim. 2009; Whiteman, Zeiders, Killoren, Rodríguez, \& Updegraff, 2014). La mayor parte de los artículos revisados muestran una tendencia a ubicar y describir esta teoría como marco teórico de su investigación, pero no se ocupan de evaluar la teoría misma en relación al aprendizaje de comportamientos sexuales entre hermanos. Existe una minoría de estudios que buscan comprender este proceso de influencia fraterna en el comportamiento sexual a partir de la teoría del guion sexual -un artículo- (Kornreich, Hearn, Rodríguez, \& O'Sullivan, 2003) y de la teoría de la comparación social -un artículo- (Haurin, \& Mott, 1990).

Finalmente, es importante destacar que en el entendimiento de las estrategias de socialización sexual fraterna y en la influencia que tienen los hermanos en el comportamiento sexual, existen diversos estudios (Haurin, \& Mott, 1990; East, Felice, \& Morgan, 1993; East, 1996; Widmer, 1997; Olenick, 1998; East, \& Jacobson, 2001; Klein, Graff, Green \&\& Kodjo, 2003; Rucibwa, Modeste, Montgomery, \& Fox, 2003; Diop-Sidibé, 2005; East, Reyes, \& Horn, 2007; Borges, Latorre, \& Schor, 2007) que marcan una tendencia de conceptuar a los hermanos mayores como agentes "patógenos" o "de influencia negativa" sobre el comportamiento sexual de los hermanos menores; sin embargo, son escasas las investigaciones que buscan determinar los aspectos de influencia positiva para prevenir algún problema de salud sexual (Kornreich, Hearn, Rodríguez, O'Sullivan, 2003; Kowal, \& Blinn-Pike, 2004).

b) Influencia de los hermanos sobre el comportamiento sexual de riesgoy prevención

Gran parte de la investigación especializada que se ha dirigido a estudiar cómo los hermanos influyen en el comportamiento sexual de riesgo, ha destacado que la condición de tener "un hermano/a mayor adolescente con hijos" es un factor que predispone en la interacción fraterna de ciertas variables psicológicas. El hecho de tener un hermano/a con hijos, si bien no es una variable psicológica, si puede ser considerada una variable de la estructura familiar. Miller, Forehand, \& Kotchick (1999) señalan que existen variables que son propias de la estructura familiar -de orden sociodemográfico- y que pueden tener impacto en variables psicosociales y conductuales asociadas al comportamiento sexual de riesgo o preventivo.

Por ejemplo, en diversos estudios se ha descrito cómo los adolescentes que tienen experiencia sexual y que juegan el rol de hermanos menores (Rucibwa, et al., 2003; Klein, et al., 2003; Diop-Sidibé, 2005), iniciaron su debut sexual a edades tempranas (East, et al., 1993; Borges, Latorre, \& Schor, 2007), no usan píldoras anticonceptivas o condón para protegerse durante el coito (Klein, et al., 2003) e informan de un incremento en la frecuen- cia de la actividad sexual y el número de parejas sexuales (East, \& Jacobson, 2001); son quienes reportan tener un hermano/a adolescente con hijos -en su rol de hermano mayor-. Además, la interacción con los hermanos en esta condición incrementa en las mujeres las posibilidades de haber estado embarazada alguna vez (East, \& Jacobson, 2001; Klein, et al., 2003; East, et al., 2007).

Los resultados citados anteriormente muestran que existen condiciones de la estructura familiar-como tener o no un hermano/a mayor adolescente con hijos- que aparentemente tienen una influencia sobre el comportamiento sexual de riesgo; sin embargo, se requiere comprender cómo es que estas variables estructurales -como las Ilaman Miller, et al. (1999)- terminan influyendo en el comportamiento sexual de riesgo. En este proceso de comprensión entre la estructura familiar y el comportamiento sexual de los hermanos es donde se han sugerido explicaciones a través de variables psicosociales como las creencias y actitudes.

Por ejemplo, cuando se tiene un/a hermano/a adolescente mayor con hijos se refuerzan creencias como "tener un hijo no implica un problema para terminar de cursas los estudios, debido a que se puede contar con la familia" (Klein, et al., 2003) e "iniciar la vida sexual a edades tempranas está bien" (East, 1996). Asimismo se fomentan actitudes sexuales permisivas, intenciones sobre tener actividad sexual en el futuro (East, et al., 1993) y de querer tener un hijo; además de producir un efecto de receptividad y actitud positiva ante la maternidad (East, 1996).

Este tipo de creencias, intenciones y actitudes promueven comportamientos sexuales de riesgo, y a través de estas variables se puede entender como la estructura familiar termina impactando en el comportamiento sexual de riesgo de los hermanos menores. En conclusión, Diop-Sidibé (2005) señala que el hermano menor percibiría la parentalidad del hermano mayor como una condición beneficiosa, por lo que podría ser animado a participar en la actividad sexual y tener hijos.

Por otro lado, existe otro grupo de estudios que evalúan la influencia de los hermanos mayores pero sin la condición de ser padres adolecentes. Estos estudios están centrados en evaluar variables psicosociales (creencias y percepción de la cercanía fraterna) y conductuales (actividad sexual, frecuencia de las relaciones sexuales, etcétera), más que factores propios de la estructura familiar, para explicar la influencia fraterna en el comportamiento sexual de riesgo.

Por ejemplo, Olenick (1998) evaluó adolescentes sexualmente activos -con el rol de hermano menor- y comparó dos grupos: quienes tenían hermanos mayores con experiencia sexual y quienes los tenían sin experien- 
cia sexual. En los resultados describe una diferencia significativa en ambos grupos, siendo mayor la proporción de estudiantes quienes informaron que sus hermanos mayores eran sexualmente activos. Al evaluar las creencias de los hermanos mayores se encontró que es significativamente mayor el porcentaje de hermanos menores sexualmente activos que tenían hermanos mayores quienes creían que "es aceptable iniciar la vida sexual antes de los 17 años" en comparación con quienes creían que era "mejor esperar más tiempo para debutar sexualmente". Los resultados citados anteriormente muestran que las creencias de los hermanos mayores sexualmente activos tienen un impacto sobre el hecho de que los hermanos menores tengan experiencia sexual.

Igual que las creencias, la percepción de la cercanía fraterna es otro factor psicosocial que mediará algunas variables asociadas al comportamiento sexual de los hermanos. Por ejemplo, de acuerdo a los resultados expuestos por Kowal, \& Blinn-Pike (2004) existe una estrecha relación entre la percepción de la cercanía fraterna y la frecuencia de las discusiones sobre sexo entre hermanos. Los resultados mostraron que a mayor frecuencia de la comunicación sexual fraterna, mayor nivel de auto eficacia para obtener y usar condones.

Sin embargo, cabe destacar que además de las creencias y la percepción de la cercanía fraterna, la conducta sexual de los hermanos mayores -variable conductual- también es una variable a considerar. Widmer (1997) destaca la importancia que tienen los comportamientos sexuales de los hermanos mayores sobre la conducta de tener la primera relación sexual en los hermanos menores. En su investigación, la actividad sexual de los hermanos mayores se torna un predictor -junto con algunas variables parentales- de la edad del debut sexual de los hermanos menores, resultado que se torna consistente con los hallazgos expuestos por Haurin, \& Mott (1990).

Para finalizar, es importante subrayar que dentro del análisis de la influencia fraterna en los comportamientos sexuales de riesgo y prevención, existen resultados diferenciados por sexo. De acuerdo con Widmer (1997), se pueden generar confusiones en la interpretación de los resultados si no se consideran las posibles combinaciones de diadas -mismo sexo o mixtas (hombre/mujer)- Además, es fundamental la inclusión del género debido a que las expectativas socioculturales que se tienen de hombres y mujeres son diferentes (Rocha-Sánchez, \& Díaz-Loving, 2005), por tanto, la socialización sexual fraterna, dependerá en algún grado de los estereotipos y la construcción de género (Kornreich, et al., 2003; Rani, et al., 2003). Bajo este supuesto, se ha descrito que existe mayor influencia entre las diadas del mismo sexo (Widmer, 1997; Diop-Si- dibé, 2005) que en diadas mixtas, pero principalmente en diadas de mujeres (East \& Jacobson, 2001; Borges, et al., 2007; Kowal, \& Blinn-Pike, 2004) en comparación con las diadas de varones (Widmer, 1997).

La explicación para estos resultados se basa principalmente en los estereotipos de género. De acuerdo con Brody (1998) en las diadas fraternas del mismo sexo se genera mayor compatibilidad, calidez y cercanía, por tanto, esta condición afectiva genera una relación más sólida y en consecuencia se incrementa la probabilidad de que se lleve a cabo la imitación del hermano menor y el modelado del hermano mayor (Brody, 1998; Buist, et al., 2013). Sin embargo, principalmente las diadas fraternas del sexo femenino se caracterizan por ser intensas en el compañerismo e intimidad, a diferencia de las díadas de hermanos varones y mixtas (Spitze, \& Trent, 2006); inclusive, en el caso de las mujeres, las relaciones estrechas no solamente permiten el modelado de la hermana mayor hacia la menor, sino también se torna como una condición que facilita la comunicación sexual fraterna (Kowal, \& Blinn-Pike, 2004). Lo anterior cobra sentido si se considera el impacto que tiene el estereotipo de género femenino, el cual se torna descriptivo y prescriptivo de lo que se espera a nivel sociocultural de las mujeres en una relación, es decir, la expectativa sobre ellas en comparación de ellos, es que ejerzan un rol afectivo y emocional en el momento de relacionarse con otros (Rocha-Sánchez, \& Díaz-Loving, 2005).

\section{DisCusión}

La presente revisión analiza y organiza diversas evidencias empíricas -publicadas en los últimos 14 añospara argumentar la importancia que tienen los hermanos en el proceso de socialización sexual, con el objetivo de reconocer e incluir al grupo de hermanos junto con los padres, en el análisis del comportamiento sexual preventivo y de riesgo, de tal manera que se brinde una perspectiva más amplia sobre el complejo proceso de socialización sexual que brinda la familia.

Los resultados expuestos en esta revisión muestran que los hermanos mayores se constituyen como agentes activos en el proceso de socialización sexual de los hermanos menores ya sea de forma directa a través de la comunicación sexual fraterna, discutiendo y brindando información sobre diversos tópicos relativos al sexo, o de manera indirecta a través de sus comportamientos, actitudes y creencias. Estas estrategias de socialización pueden tener impacto en los hermanos menores, si se considera que en países donde se fomentan los valores colectivistas como en México, la responsabilidad de 
cuidar a los hermanos pequeños se delega a un hermano/a mayor cuando ambos padres se tienen que ausentar por cuestiones laborales o personales, o cuando éstos son negligentes (Goetting, 1986), por tanto, los hermanos mayores se convierten en cuidadores secundarios y adquieren poder y responsabilidad sobre el cuidado y control de los hermanos pequeños (Cicirelli, 1994), circunstancia que los pone en una posición "similar" a la de los padres, quienes son los agentes primarios de la socialización sexual de acuerdo con Widmer (1997).

Aun así, cabe destacar que el papel de los hermanos en el proceso de socialización sexual pudiera ser más relevante en las culturas latinas que en otras. En el contexto de la familia mexicana al igual que en las familias latinas con valores colectivistas (Triandis, 2006; Triandis, \& Suh, 2002), se premian cualidades como la cooperación, la cercanía, la lealtad o el auto sacrificio; se esperaría que las relaciones entre hermanos fueran más intensas y con mayor matiz afectivo que en culturas donde se promueven valores individualistas. Este último punto adquiere importancia si se considera que de las 20 investigaciones analizadas para el presente estudio, solamente una se realizó con población mexicana y dos más con muestras de hermanos provenientes de familias latinas. Por tanto, se requiere generar más investigación en México y Latinoamérica debido a que las características de las familias mexicanas y americanas tienen importantes diferencias en los patrones de comportamiento y de socialización (Díaz-Guerrero, \& Szalay, 1993).

Asimismo, para continuar avanzando en el estudio de la influencia fraterna sobre el comportamiento sexual, es importante atender a las críticas metodológicas en torno a la evaluación de este fenómeno. McHale, et al. (2009) y Whiteman, et al. (2014) señalan que cuando se pretende evaluar la influencia fraterna a través de la teoría del aprendizaje social -la cual resultó ser la más utilizada para este fenómeno-, en realidad no se evalúa de forma directa, por el contrario, esta teoría se utiliza principalmente como una explicación post hoc para describir los patrones observados de similitudes y diferencias entre hermanos. La recomendación es obtener medidas directas de los procesos de modelización -modelado, imitación, etcétera- en lugar de inferir sobre ellos y sobre el funcionamiento en el contexto de la relación entre hermanos (Whiteman, et al., 2014).

Ahora, la teoría del aprendizaje social no es la única aproximación posible que existe para dar cuenta de cómo y bajo qué circunstancias los hermanos mayores pudieran influir en los comportamientos sexuales de los menores. Existen otras propuestas como la teoría de la comparación social (Haurin, \& Mott, 1990), la teoría de los guio- nes sexuales (Kornreich, et al., 2003) y la teoría del grupo de referencia (Widmer, 1997), para comprender y explicar este proceso de influencia fraterna. Asimismo, existen otros procesos aparte de la imitación y la observación que podrían ser considerados en la comprensión de este fenómeno; por ejemplo, la de identificación o diferenciación fraterna (Whiteman, et al., 2014) o los estilos de cuidado y protección que los hermanos brindan (siblings gatekeeping) (Kornreich, et al., 2003).

Cabe destacar que independientemente de la teoría utilizada y de los factores psicosociales que se busquen investigar en torno a cómo los hermanos influyen en el comportamiento sexual, es importante comenzar a describir aquellas variables psicológicas más que estructurales que permitan predecir comportamientos sexuales preventivos o una conducta sexual protegida (Kornreich, Hearn, Rodríguez, \& O’Sullivan, 2003; Kowal, \& Blinn-Pike, 2004). Como se señaló en el análisis de resultados, existe una tendencia a conceptuar a los hermanos mayores como agentes "patógenos" o "de influencia negativa" sobre el comportamiento sexual de los hermanos menores; sin embargo, en la medida en que se comience a vislumbrar el potencial que tienen los hermanos como agentes promotores de la salud sexual, los esfuerzos se pueden dirigir a la identificación de aquellas variables que hagan más o menos probable que los hermanos mayores influyan sobre los menores, a través de sus comportamientos, actitudes, intenciones o creencias, para que éstos utilicen por ejemplo, el condón en sus relaciones sexuales.

De acuerdo a la revisión realizada, se espera que los hermanos mayores sean quienes influyan en los menores, se sabe poco sobre si los hermanos menores pueden tener influencia sobre el comportamiento sexual de los mayores. Lo anterior abre una línea de investigación donde se requiere aportar evidencia empírica para llegar a una conclusión sólida. Si bien hay estudios que señaIan la posible existencia de algún tipo de influencia(Rodgers, Rowe, \& Harris, 1992; Whiteman, \& Christiansen, 2008), se carece de teorías para explicar este proceso; en consecuencia, es necesario desarrollar investigación para delinear y describir las reglas y procesos bajo los cuales los hermanos menores influyen sobre el comportamiento sexual de los mayores -si es que esto es posible y se justifica con mayor evidencia empírica- ya que la investigación existente no es concluyente.

Este último punto es importante porque al analizar el rol que juegan los hermanos mayores y menores, necesariamente se tiene que discutir el papel de la cultura. La expectativa cultural que se tiene de los hermanos mayores es que deben ser como maestros, mentores y 
amigos de los hermanos menores que requieren cuidados (Craft-Rosenberg, et al. 2011; Enríquez, Arias, Sánchez, \& Robles, 2011). En este sentido la cultura brinda demandas complementarias entre el rol del hermano mayor y menor. Bajo este contexto, se puede tener una posible explicación complementaria sobre por qué los hermanos menores que requieren ser cuidados siguen a los mayores en su rol de maestros o mentores y aprenden de ellos al mismo tiempo que son influidos.

Este análisis se torna más complejo cuando se incluye el género en el análisis de las diadas, de tal forma que se cruza el rol fraterno -hermano mayor/menor- con el género. Los estereotipos de género aportan información relevante sobre las expectativas, normas y cosmovisiones que existen alrededor de hombres y mujeres en una cultura determinada. En este sentido, en la cultura mexicana se espera que la mujer ejerza un rol afectivo centrado en las actividades relativas del cuidado de los otros; en el caso de los hombres, se espera el ejercicio de un rol instrumental caracterizado por la autonomía, el logro y el éxito pero no por la expresión del afecto y las emociones (Rocha-Sánchez, \& Díaz-Loving, 2005).

Bajo este contexto, el rol fraterno como hermano mayor o menor puede cobrar fuerza y afianzarse a partir de las expectativas sociales y culturales del género. De esta forma, se podría tener por ejemplo, diadas de hermanas mayores quienes se comportan como guías y mentoras de sus hermanas pequeñas, con el propósito de prevenirlas y cuidarlas de un riesgo sexual potencial, lo cual podría favorecer ciertas actitudes y comportamientos que pudieran promover valores tradicionales del rol femenino como la castidad. Sin embargo, también podría suceder lo contrario, es decir, promover valores tradicionales del rol femenino como la maternidad, lo cual pudiera tener relación con la intención de querer un bebé en lo inmediato (East, \& Jacobson, 2001) o en la receptividad y actitud positiva ante la maternidad (East, 1996).

Otro ejemplo podría ser respecto al rol del hermano mayor cuando éste se ejerce con hermanos menores varones. Por expectativa cultural, se espera que los hombres busquen refrendar su libertad sexual frente a las mujeres, que tengan múltiples conquistas amorosas y que aprovechen cualquier oportunidad de relacionarse sexualmente para obtener aprendizaje en el terreno sexual (Marin, 1989; Wood, \& Price, 1997)-dimensión de género. Cuando se vinculan estas expectativas con el rol fraterno del hermano mayor, se podría comprender porque los hermanos mayores al ser percibidos como mentores y maestros (Craft-Rosenberg, et al., 2011) podrían instruir y fomentar en los hermanos menores iniciar la vida sexual a edades tempranas o promover la actividad sexual (Haurin, \& Mott, 1990; Olenick, 1998; Widmer, 1997).

Ambos ejemplos permiten ilustrar cómo los factores culturales reflejados en el rol fraterno y de género, se encuentran estrechamente marcados en las relaciones entre hermanos. Además de las estrategias de socialización sexual, de las variables de estructura familiar y psicosociales, de las teorías explicativas sobre la influencia fraterna; los aspectos culturales no pueden pasarse por alto en la explicación y comprensión del papel que tienen los hermanos en los diversos comportamientos sexuales de riesgo y preventivos.

\section{ConcLusiones}

La presente revisión muestra a través del análisis de la evidencia empírica, que los hermanos a pesar de fungir como fuentes secundarias de socialización sexual después de los padres, pueden tener un impacto tanto positivo como negativo en los conocimientos, actitudes, intenciones y valores de la sexualidad. Cabe destacar que es necesario continuar investigando en qué circunstancias y cómo es que se produce este proceso de influencia fraterna sobre el comportamiento sexual. Por tanto, es necesario desarrollar algunas consideraciones para investigaciones futuras.

Por ejemplo, para seguir avanzando en la descripción y comprensión del tema es necesario: a) incluir en el análisis de las relaciones fraternas, tanto variables de estructura familiar (por ejemplo "tener un/a hermano/a adolescente con hijos") como variables conductuales (lo que los hermanos hacen) y psicosociales (creencias y actitudes relativas al sexo); b) no suponer influencia fraterna a partir de las similitudes y diferencias entre hermanos utilizando explicaciones post hoc, como por ejemplo la teoría del aprendizaje social que es la que mayormente se utiliza en este campo, sino por el contrario, evaluar y obtener datos del mismo proceso de influencia, (por ejemplo; modelado, imitación, cercanía fraterna, etcétera) para determinar el impacto que tiene la teoría en la explicación de la influencia fraterna; c) generar investigación que busque aportar conclusiones sólidas sobre si existe o no una influencia de los hermanos menores hacia los mayores y desarrollar teorías que permitan explicar y comprender dicho fenómeno; d) analizar el papel positivo que los hermanos tienen sobre el comportamiento sexual preventivo y considerar la posibilidad de utilizarlos como agentes para modelar comportamientos preventivos en los hermanos menores (por ejemplo, respecto a cómo usar correctamente un condón, cómo iniciar una conversación con la pareja 
sobre sexo, cómo negociar el preservativo, etcétera), pero también pueden constituirse como agentes que puedan informar correctamente a los hermanos menores e incidir sobre sus creencias y actitudes en torno a la prevención de las ITS, el embarazo no deseado y por supuesto el VIH/SIDA. Este cambio de percepción de los "hermanos nocivos para la salud sexual" hacia una perspectiva de los "hermanos como promotores de la salud sexual" implica llevar a cabo un trabajo en el cual se informe correctamente, entrene y sensibilice a los hermanos mayores sobre el impacto que pueden tener sobre el comportamiento sexual de sus hermanos menores; y e) se sugiere llevar a cabo estudios donde sea posible hacer la comparación entre diadas por rol fraterno (mayor/menor) y sexo (diadas mixtas o del mismo sexo) tomando en cuenta que tanto el rol fraterno como el de género deben ser interpretados desde las expectativas y prescripciones culturales.

Por último, es importante señalar que los hermanos pueden tener una contribución moderada o secundaria dentro de la socialización sexual fraterna; por supuesto esto puede variar a partir de la estructura familiar, del tipo de relaciones familiares que se establezcan, de la etapa del ciclo vital en la cual se encuentre la familia y los hermanos, de la edad de los hermanos, el sexo y por supuesto de la cultura. Por tanto, la idea central es destacar el papel y la contribución que pudieran tener los hermanos en relación a los padres. Analizar la influencia familiar en el comportamiento sexual de riesgo implicaría entonces no solamente centrarse en los padres, sino incluir tanto variables parentales como fraternas en la predicción y explicación del comportamiento sexual preventivo y de riesgo.

\section{Referencias}

American Psychological Association. (2014). PsycINFO. Recuperado de http://www.apa.org/pubs/databases/psycinfo/

Bárcena, G. S., Rendón, R. G., \& Robles, M. S. (2011). Factores asociados a la conducta sexual protegida: una revisión. En R. Díaz-Loving, \& S. Robles Montijo (Eds.), Salud y Sexualidad. México D.F.: UNAM, FES Iztacala, Asociación Mexicana de Psicología Social, Consejo Nacional de Ciencia y Tecnología, 5-47.

Bastien, S., Kajula, L. J. \& Muhwezi, W. W. (2011). A review of studies of parent-child communication about sexuality and HIVIAIDS in sub-Saharan Africa. Reproductive Health, 8(1), 1-17.

Borges, A. L., Latorre, M. R., \&. Schor, N. (2007). Factors associated with sexual debut among adolescents enrolled in a family health unit in East Side São Paulo, Brazil. Cadernos de saúde pública,(23)7, 1583-1594.
Brody, G. H. (1998). Sibling relationship quality: Its causes and consequences. Annual Review of Psychology, 49, 1-24.

Buist, K. L., Deković, M., \& Prinzie, P. (2013). Sibling relationship quality and psychopathology of children and adolescents: A meta-analysis. Clinical Psychology Review, 33(1), 97-106.

Cicirelli, V. G. (1994). Sibling relationships in cross-cultural perspective. Journal of Marriage and Family, 56(1), 7-20.

Consejo Nacional para la Prevención y Control del SIDA (CENSIDA, 2011). EI VIH/SIDA en México 2011. Recuperado de

http://www.censida.salud.gob.mx/descargas/2011/ vihsidaenmexico2011.pdf

Consejo Nacional para la Prevención y Control del SIDA (CENSIDA, 2012). Vigilancia epidemiológica de casos de VIH/SIDA en México. Registro Nacional de Casos de SIDA. Actualización al 30 de Junio del 2012. Recuperado de http://www.censida.salud.gob.mx/descargas/2012/sida vih30junio2012pub2.pdf

Craft-Rosenberg, M., Montgomery, L. A., Hill, J., Kauder, J., \& Eisbach, S. (2011). Sibling death/loss. En M. CraftRosenberg, \& P. Shelley-Rae Pehler (Eds.), Encyclopedia of Family Health (pp. 951-957). Thousand Oaks, USA: SAGE Publications.

Davis, E. C., \& Friel, L. V. (2001). Adolescent sexuality: disentangling the effects of family structure and family context. Journal of Marriage and Family, 63(3), 669-681.

Díaz-Guerrero, R., \& Szalay, L. B. (1993). El mundo subjetivo de mexicanos y norteamericanos. México, D.F.: Trillas.

Diop-Sidibe, N. (2005). Siblings' premarital childbearing and the timing of first sex in three major cities of Cote d'Ivoire. International Family Planning Perspectives, 31(2), 54-62.

East, P. L., \& Jacobson, L. J. (2001). The younger siblings of teenage mothers: a follow-up of their pregnancy risk. Developmental psychology, 37(2), 254-264.

East, P. L. (1996). Do adolescent pregnancy and childbearing affect younger siblings? Family Planning Perspectives, 28(4), 148-153.

East, P. L., Reyes, B. T., \& Horn, E. J. (2007). Association between adolescent pregnancy and a family history of teenage births. Perspectives on Sexual and Reproductive Health, 39(2), 108-115.

East, P. L., Felice, M. E., \& Morgan, M. C. (1993). Sisters' and girlfriends' sexual and childbearing behavior: Effects on early adolescent girls' sexual outcomes. Journal of Marriage and Family, 55(4), 953-963.

East, P., \& Khoo, S. (2005). Longitudinal pathways linking family factors and sibling relationship qualities to adolescent substance use and sexual risk behaviors. Journal of Family Psychology, 19 (4), 571-580.

EISevier (2011). ScienceDirect. Recuperado de http://www.americalatina.elsevier.com/corporate/es/ centro de estudos.php

Encuesta Nacional de Salud y Nutrición (ENSANUT, 2012). Encuesta Nacional de Salud y Nutrición. Resultados Nacionales 2012. Recuperado de

h t t p: / / ensanut.ins p. mx/informes/ ENSANUT2012ResultadosNacionales.pdf 
Enríquez, D. (2014). Papel del contexto familiar en la conducta sexual protegida de jóvenes universitarios: una perspectiva sistémica. Tesis de Doctorado no publicada, Facultad de Estudios Superiores Iztacala, Universidad Nacional Autónoma de México, Edo. De México, México

Enríquez, D., Arias, B., Sánchez, R., \& Robles, S. (junio, 2011). Significado de las relaciones fraternas: Rol de los hermanos mayores y menores. Trabajo presentado en el XXXIII Congreso Interamericano de Psicología, Medellín, Colombia. Memorias en extenso, 1249.

Enríquez, D., Sánchez, R., \& Robles, S. (mayo, 2011). Construcción de una Escala de Relaciones Fraternas: un estudio exploratorio. Trabajo presentado en el $8^{\circ}$ Coloquio Nacional y $2^{\circ}$ Internacional de Investigación Estudiantil en Psicología, Estado de México, México.

Feinberg, M. E., \& Hetherington, E. M. (2000). Sibling differentiation in adolescence: Implications for Behavioral Genetic Theory. Child development, 71(6), 1512-1524.

Feinberg, M. E., Sakuma, K. L., Hostetler, M., \& McHale, S. M. (2013). Enhancing sibling relationships to prevent adolescent problem behaviors: Theory, design and feasibility of siblings are special. Evaluation and Program Planning, 36(1), 97-106.

Goetting, A. (1986). The developmental tasks of siblingship over the life cycle. Journal of Marriage and Family, 48(4), 703714.

Haurin, R. J., \& Mott, F. L. (1990). Adolescent sexual activity in the family context: The impact of older siblings. Demography, 27(4), 537-557.

Hovell, M., Sipan, C., Blumberg, E., Atkins, C., Hofstetter, C. R., \& Kreitner, S. (1994). Family Influences on Latino and Anglo Adolescents' Sexual Behavior. Journal of Marriage and Family, 56(4), 973-986.

JSTORE (2014). New to JSTOR? Learn more about us. Recuperado de http://about.jstor.org/10things

Klein, J. D., Graff, C. A., Green, A., \& Kodjo, C. (2003). Adolescent pregnancy prevention: Impact of siblings who are teen parents. Journal of Adolescent Health, 32(2), 134-135.

Kornreich, J. L., Hearn, K. D., Rodriguez, G., \& O'Sullivan, L. F. (2003). Sibling influence, gender roles, and the sexual socialization of urban early adolescent girls. The Journal of Sex Research, Gender and Sexuality, 40(1), 101-110.

Kotchick, B. A., Shaffer, A., Miller, K. S., \& Forehand, R. (2001). Adolescent sexual risk behavior: a multi-system perspective. Clinical Psychology Review, 21(4): 493-519.

Kowal, A. K., \& Blinn-Pike, L. (2004). Sibling influences on adolescents' attitudes toward safe sex practices. Family Relations, 53(4), 377-384.

Lucey, H. (2010). Families, siblings and identities. En M. Wetherell, \& C. T. Mohanty (Eds.), The Sage Handbook of Identities, (pp. 476-491). Ltd. London, UK: SAGE Publications, Ltd.

Manlove, J., Wildsmith, E., Ikramullah, E., Terry-Humen, E., \& Schelar, E. (2012). Family environments and the relationship context of first adolescent sex: Correlates of first sex in a casual versus steady relationship. Social Science Research, 41(4), 861-875.

Marín, G. (1989). AIDS prevention among Hispanics: Needs, risk behaviors, and cultural values. Public Health Reports, 105(5), 411-415.
Martino, S. C., Elliott, M. N., Corona, R., Kanouse, D. E., \& Schuster, M. A. (2008). Beyond the "Big Talk": The roles of breadth and repetition in parent-adolescent communication about sexual topics. Pediatrics, 121(3), 612-618.

McHale, S. M., Bissell, J., \& Kim, J.-Y., (2009). Sibling relationship, family, and genetic factors in sibling similarity in sexual risk. Journal of family psychology, 23(4), 562-572.

Miller, K. S., Forehand, R., \& Kotchick, B. A. (1999). Adolescent sexual behavior in two ethnic minority samples: the role of family variables. Journal of Marriage and Family, 61(1), 85-98.

Noar, S. M. (2007). An interventionist's guide to AIDS behavioral theories. AIDS Care - Psychological and Socio-medical Aspects of AIDS/HIV, 19(3), 392 - 402.

Olenick, I. (1998). Adolescents with sexually active older siblings are likely to have sex early. Family Planning Perspectives, 30(3), 149-150.

Oliveira-Campos, M., Giatti, L., Malta, D., \& Barreto, S. M. (2013). Contextual factors associated with sexual behavior among Brazilian adolescents. Annals of Epidemiology, 23(10), 629-635.

Rani, M., Figueroa, M. L., \& Ainsle, R. (2003). The psychosocial context of young adult sexual behavior in Nicaragua: Looking through the gender lens. International Family Planning Perspectives, 29(4), 174-181.

Rittenour, C. E., Myers, S. A., \& Brann, M. (2007). Commitment and emotional closeness in the sibling relationship. Southern Communication Journal, 72(2), 169-183.

Rocha-Sánchez T., \& Díaz-Loving, R. (2005). Cultura de género: La brecha ideológica entre hombres y mujeres. Anales de Psicología, 21(1), 42-49.

Rodgers, J. L., Rowe, D. C., \& Harris, D. F. (1992). Sibling differences in adolescent sexual behavior: inferring process models from family composition patterns. Journal of Marriage and Family, 54(1), 142-152.

Rucibwa, N. K., Modeste, N., Montgomery, S., \& Fox, C. (2003). Exploring family factors and sexual behaviors in a group of Black and Hispanic adolescent males. American journal of health behavior, (27)1, 63-74.

Secretaría de Salud (2002). Programa de acción: VIH/SIDA e Infecciones de Transmisión Sexual (ITS). Recuperado de http://www.salud.gob.mx/docprog/estrategia_3/sida.pdf

Spitze, G., \& Trent, K. (2006). Gender differences in adult sibling relations in two-child families. Journal of Marriage and Family, 68(4), 977-992.

Triandis, H. C. (2006). Cultural aspects of globalization. Journal of International Management, 12(2), 208-217.

Triandis, H. C., \& Suh, E. M. (2002). Cultural influences on personality. Annual Review of Psychology, 53(1), 133-160.

United States National Library of Medicine (2014). Leasing Diario Citaciones (MEDLINE/PubMed). Recuperado de http://www.nlm.nih.gov/databases/journal.html

Upchurch, D. M., Aneshensel, C. S., Sucoff, C. A., \& Levy-Storms, L. (1999). Neighborhood and family contexts of adolescent sexual activity. Journal of Marriage and the Family, 61(4), 920-933.

Uribe, J., I. (2007). Factores individuales, sociales y culturales asociados a la conducta sexual de adolescentes (Tesis Doctoral inédita). Facultad de Psicología, Universidad 
Nacional Autónoma de México, Distrito Federal, México.

Vélez-Pastrana, M., C., González-Rodríguez, R. A., \& BorgesHernández, A. (2005). Family functioning and early onset of sexual intercourse in latino adolescents. Adolescence, 40(160), 777-791.

Vukovic, D. S., \& Bjegovic, V. M. (2007). Brief report: Risky sexual behavior of adolescents in Belgrade: Association with socioeconomic status and family structure. Journal of Adolescence, 30(5), 869-877.

Wallace, S. A. (2008). 63: I am my brother's keeper: Sibling influences on sexual attitudes and behaviors among urban black youth, Journal of Adolescent Health, 42(2), 43.

Ward, L. M. (2003). Understanding the role of entertainment media in the sexual socialization of American youth: $A$ review of empirical research. Developmental Review, 23(3), 347-388.
Whiteman, S. D, Zeiders, K. H., Killoren, S. E, Rodriguez, S. A., \& Updegraff, K. A. (2014). Sibling influence on Mexicanorigin adolescents' deviant and sexual risk behaviors: The role of sibling modeling. Journal of Adolescent Health, 54(5), 587-592.

Whiteman, S. D. \& A. Christiansen (2008). Processes of sibling influence in adolescence: individual and family correlates. Family Relations, 57(1), 24-34.

Widmer, E. D. (1997). Influence of older siblings on initiation of sexual intercourse. Journal of Marriage and Family, 59(4), 928-938.

Wood, M. L., \& Price, P. (1997). Machismo and marianismo: Implications for HIV/AIDS risk reduction and education. American Journal of Health Studies, 13(1), 44-52.

Ybarra, M. L, Emenyonu, N., Nansera, D., Kiwanuka, J., \& Bangsberg, D. R. (2008). Health information seeking among Mbararan adolescents: Results from the Uganda Media and you survey. Health education research, 23(2), 249-258. 
Revista Digital Internacional de Psicología y Ciencia Social $\mid$ Vol. $2 \mid$ Núm. $1 \mid$ Enero-Junio 2016 | ISSN 1405-5802

\section{Dimensión Cuantitativa}

\section{Perfil de Evaluación entre pares}
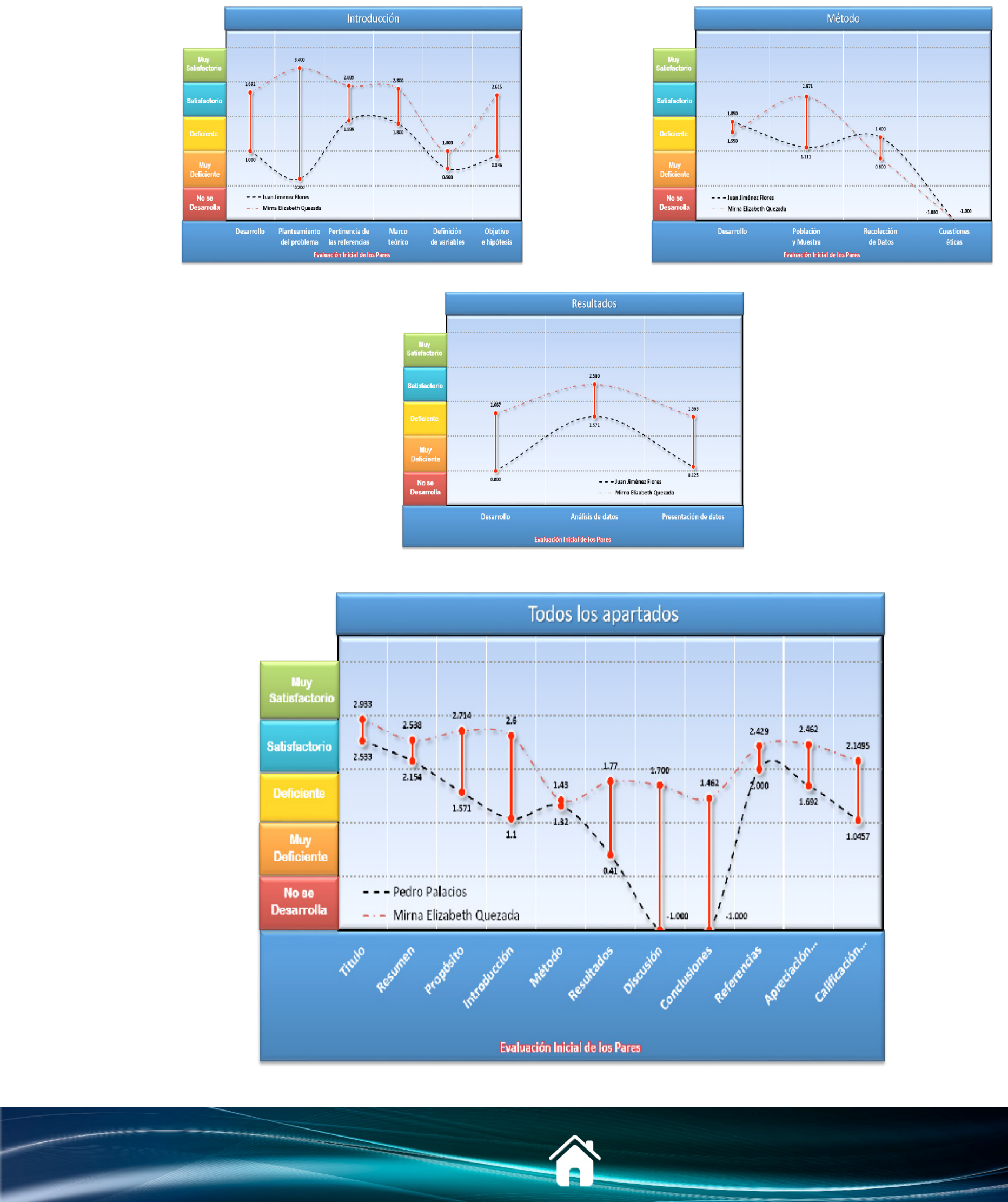
Revisión de literatura | Influrncia de los hermanos en el comportamiento sexual... | Enriquez-Negrete dZ Sánchez-Medina

\section{Índice de Concordancia}

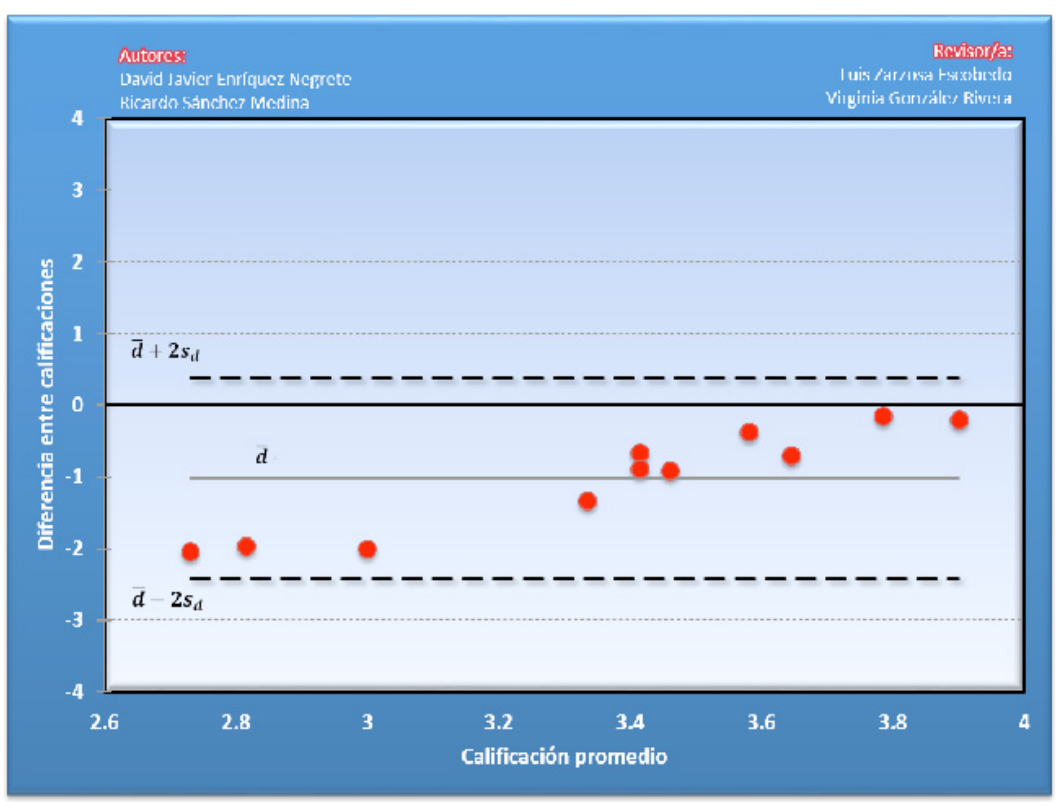

Índice de Acuerdo
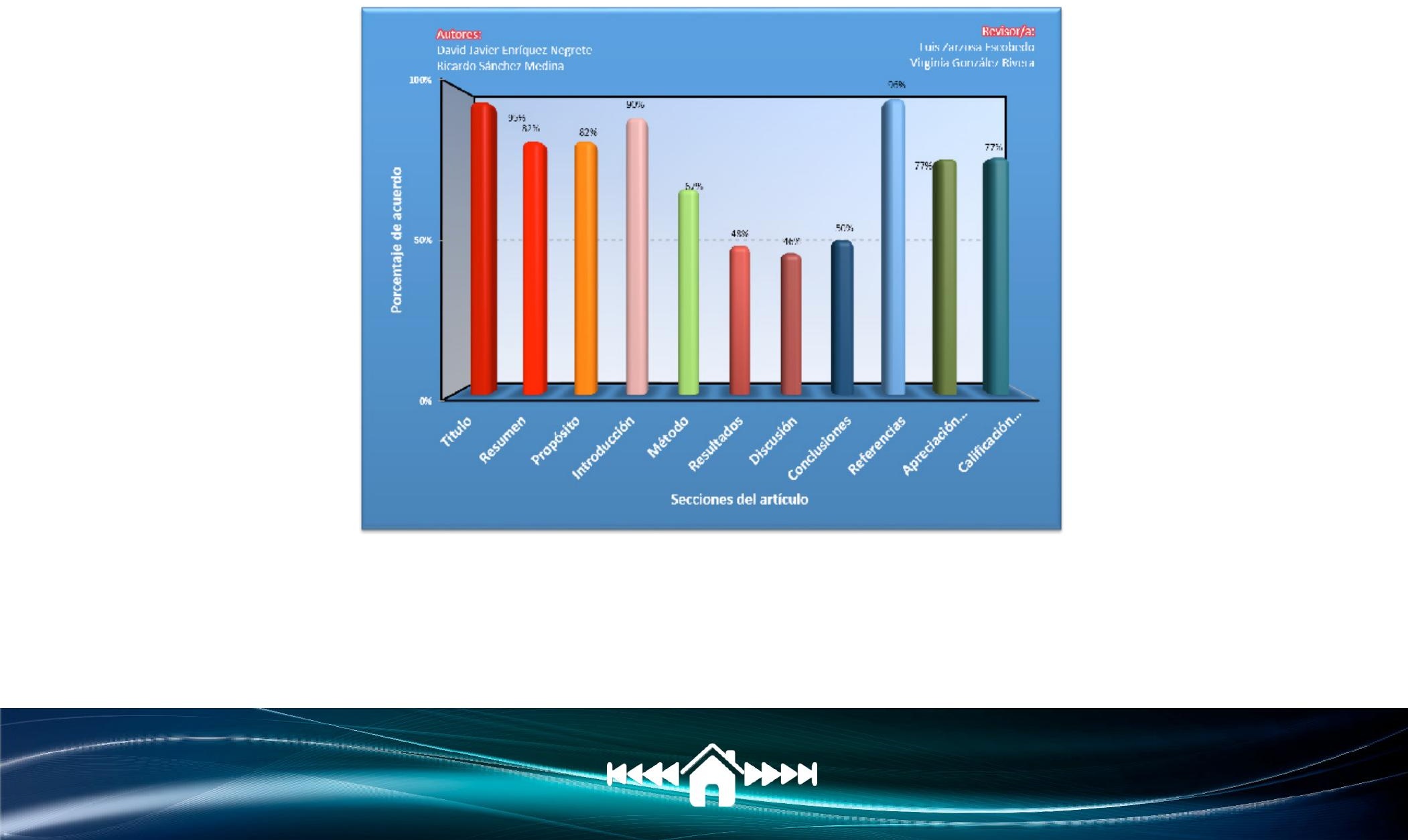


\begin{tabular}{|c|c|}
\hline Revisor 1 & Revisor 2 \\
\hline Luis Zarzosa-Escobedo & Virginia González-Rivera \\
\hline \multicolumn{2}{|c|}{ Título/Autoría } \\
\hline $\begin{array}{l}\text { Me parece apropiado. Tal vez lo de "socialización frater- } \\
\text { na" puede reemplazarse por un término más coloquial } \\
\text { como "influencia de los hermanos". Por esta razón el } \\
\text { artículo sería más atractivo e informaría de modo más } \\
\text { sencillo su contenido. }\end{array}$ & $\begin{array}{l}\text { El título me parece adecuado, yo no le haría ningun } \\
\text { cambio. }\end{array}$ \\
\hline \multicolumn{2}{|c|}{ Resumen } \\
\hline $\begin{array}{l}\text { Resulta innecesario señalar lo de la influencia del her- } \\
\text { mano menor hacia el mayor, pues simplemente es algo } \\
\text { no contemplado en los trabajos revisados; es una in- } \\
\text { quietud de los autores para la cual, en el cuerpo del es- } \\
\text { crito, no hubo suficientes argumentos para considerar- } \\
\text { la como posibilidad. Es desafortunada la expresión "no } \\
\text { puede negarse"... ¿alguien lo ha hecho? En todo caso } \\
\text { se debe argumentar la necesidad de su estudio. Si en } \\
\text { la literatura no se contempla (o mínimamente), bien } \\
\text { puede deberse a su poca trascendencia o irrelevancia. } \\
\text { En la revisión se muestra que la influencia de los her- } \\
\text { manos mayores es más bien nociva (10 trabajos vs } 2 \text {, } \\
\text { así lo indican); sin embargo esto no se contempla en } \\
\text { el resumen. Se menciona que la configuración de las } \\
\text { díadas fraternas es el punto crítico para mostrar el tipo } \\
\text { de influencia; sin embargo en la parte de "Resultados" } \\
\text { no es claro que esto haya sido así o no hay suficientes } \\
\text { trabajos para dejarlo fuera de duda; tengo la impresión } \\
\text { que es más la sospecha o la preferencia de los autores } \\
\text { que el resultado de la revisión. El punto } 7 \text { de este for- } \\
\text { mulario NO aplica para el tipo de estudio reportado. }\end{array}$ & $\begin{array}{l}\text { Quiza tenga que disminuir el número de palabras del } \\
\text { resumen e integrar la descripción de la metodología }\end{array}$ \\
\hline
\end{tabular}




\begin{tabular}{|c|c|}
\hline Revisor 1 & Revisor 2 \\
\hline \multicolumn{2}{|c|}{ Próposito del Estudio } \\
\hline $\begin{array}{l}\text { Hay problemas con el contenido de la Discusión, pues } \\
\text { se incluyen muchas consideraciones que no se derivan } \\
\text { de los resultados de la revisión. Faltó apegarse a los da- } \\
\text { tos. }\end{array}$ & Ningun comentario \\
\hline \multicolumn{2}{|c|}{ Introducción } \\
\hline $\begin{array}{l}\text { Las primeras tres o cuatro páginas del escrito resultan } \\
\text { prescindibles o deben abreviarse significativamente. } \\
\text { No se enfocan en el problema que anuncia el título. Es } \\
\text { importante establecer un deslinde: Una cosa son las } \\
\text { conductas de riesgo y/o prevención de los individuos } \\
\text { respecto a su conducta sexual, -para lo cual se consi- } \\
\text { deran sus diferentes repertorios y competencias en cir- } \\
\text { cunstancias delimitadas-, y otra muy diferente el "piso" } \\
\text { histórico de estos individuos derivado de las interac- } \\
\text { ciones formativas al interior de la familia, lo cual se } \\
\text { traduce en una tendencia o inclinación. La revisión es } \\
\text { sobre el impacto de la socialización fraterna, y hay que } \\
\text { abordar directamente el tema, sin destinar tanto espa- } \\
\text { cio (4 páginas) a consideraciones, que sin dejar de ser } \\
\text { importantes, son tangenciales. Se puede verificar cómo, } \\
\text { en la parte de Resultados, lo que se describe como las } \\
\text { diferentes variables dependientes en los estudios revi- } \\
\text { sados, no son otra cosa que inclinaciones o tendencias } \\
\text { asociadas a datos demográficos (sexo y edad de los her- } \\
\text { manos, por ejemplo). Ese es el punto medular de la re- } \\
\text { visión. Resulta problemático tratar de combinarlo con } \\
\text { los otros estudios enfocados en la parte competencial } \\
\text { específica de las conductas de riesgo/prevención. }\end{array}$ & $\begin{array}{l}\text { Falto delimitar la hipótesis o señalarla más claramen- } \\
\text { te. aunque el estudio sea una revisión se pudo haber } \\
\text { hecho redactado una hi' potesis por ejemplo, la infleu- } \\
\text { ncia fraterna determina el comportamiento sexual de } \\
\text { riesgo y prentivo en las personas. Tambien, de acuer- } \\
\text { do con mi punto de vista; aunque no se hizo una defi- } \\
\text { nición operacional de la variables, quedaron claras en } \\
\text { su definición, al leer el marco teórico, sin embargo el } \\
\text { autor pudo precisar estas. }\end{array}$ \\
\hline \multicolumn{2}{|c|}{ Método } \\
\hline $\begin{array}{l}\text { Como se trata de un trabajo de revisión, en dónde se } \\
\text { pregunta por aspectos referidos a la Población, se con- } \\
\text { sideró al número de artículos publicados como "Pobla- } \\
\text { ción". }\end{array}$ & $\begin{array}{l}\text { El método me pareció, adecuado, claro, ordenado y } \\
\text { con sufiente información opara enteder como se llevo } \\
\text { a cabo la revisión. }\end{array}$ \\
\hline
\end{tabular}


Revisor 1

\section{Resultados}

Se requiere replantear las diferentes categorías para presentar los datos de manera ordenada y enseguida ceñirse a dicho esquema. Se sugiere que las categorías se deriven de los parámetros más significativos reportados en la literatura.

\section{Discusión}

La discusión rescata los elementos teóricos y conceptuales, en las limitantes sería conveniente analizar el nivel socioeconómico de los participantes, éste puede ser un factor covariante para algunos de los hallazgos reportadas únicamente por la V.I

Aunque los resultado no estan redactados en pretérito, son pertinente por el tipo de investigación.

\section{Revisor 2}

No hay comentarios al respecto.

\section{Conclusiones}

Varias conclusiones no se justifican pues no se relacionan con los datos; otras no pasan de ser lugares comunes y alguna más no deja de ser una ocurrencia o deseo Creo que las conclusiones se abordaron de manera adecuada

\section{Referencias}

Parece todo apegado a la normatividad correspondienAunque poco menos de le $50 \%$ de las ferencias son de los últimos años son pertinentes, dado el número de referencias y el carácter del tema de investigación. 
Revisión de literatura | Influrncia de los hermanos en el comportamiento sexual... Enriquez-Negrete dZ Sánchez-Medina

\section{Historia del Proceso}

\section{EDITORIAL}

